\title{
Descriptive distribution and phylogenetic analysis of feline infectious peritonitis virus isolates of Malaysia
}

\author{
Saeed Sharif1', Siti S Arshad1*, Mohd Hair-Bejo ${ }^{1}$, Abdul R Omar ${ }^{1}$, Nazariah A Zeenathul ${ }^{1}$, Lau S Fong ${ }^{2}$, \\ Nor-Alimah Rahman ${ }^{3}$, Habibah Arshad ${ }^{2}$, Shahirudin Shamsudin², Mohd-Kamarudin A Isa ${ }^{1}$
}

\begin{abstract}
The descriptive distribution and phylogeny of feline coronaviruses (FCoVs) were studied in cats suspected of having feline infectious peritonitis (FIP) in Malaysia. Ascitic fluids and/or biopsy samples were subjected to a reverse transcription polymerase chain reaction (RT-PCR) targeted for a conserved region of $3^{\prime}$ untranslated region ( $3^{\prime} U T R$ ) of the FCoV genome. Eighty nine percent of the sampled animals were positive for the presence of FCoV. Among the FCoV positive cats, $80 \%$ of cats were males and $64 \%$ were below 2 years of age. The FCoV positive cases included 56\% domestic short hair (DSH), 40\% Persian, and 4\% Siamese cats. The nucleotide sequences of 10 selected amplified products from FIP cases were determined. The sequence comparison revealed that the field isolates had $96 \%$ homology with a few point mutations. The extent of homology decreased to $93 \%$ when compared with reference strains. The overall branching pattern of phylogenetic tree showed two distinct clusters, where all Malaysian isolates fall into one main genetic cluster. These findings provided the first genetic information of FCoV in Malaysia.
\end{abstract}

\section{Findings}

Feline infectious peritonitis (FIP) is a highly fatal disease of cats caused by generalized infection with a feline coronavirus (FCoV). FCoVs belong to subgroup 1a of Coronaviruses in the family Coronaviridae, order Nidovirales. Other members of this subgroup include porcine transmissible gastroenteritis virus, canine coronavirus, raccoon/dog coronavirus and Chinese ferret badger coronavirus $[1,2]$. FCoVs are enveloped, positive-strand RNA viruses with a large, capped and polyadenylated RNA genome of about $29 \mathrm{~kb}$. The cap structure at the 5 ' end of genome is followed by an untranslated region (UTR). At the 3' end of the genome is another UTR of 275 nucleotides, followed by the poly (A) tail. The sequences of the both 3'- and 5'-UTR are important for RNA replication and transcription [3].

Two biotypes of FCoV are described in cats: feline infectious peritonitis virus (FIPV) and feline enteric coronavirus (FECV). Infection with FECV is usually unapparent or manifested by a transient gastroenteritis. In

\footnotetext{
* Correspondence: suri@vet.upm.edu.my

'Department of Veterinary Pathology and Microbiology, Faculty of Veterinary Medicine, Universiti Putra Malaysia, 43400 UPM Serdang, Selangor, Malaysia
}

contrast, FIPV infection causes a fatal immune-mediated disease with a wide spectrum of clinical signs. FIP refers to the more common effusive (wet) form of the disease characterized by peritonitis and/or pleuritis. The effusive form is caused by complement-mediated vasculitis and results in inflammatory exudation into body cavities. In some FIP cases, partial cell-mediated immunity cause non-effusive (dry) form which is characterized by granulomatous involvement of various organs particularly central nervous system and eyes. However, the FIP forms can transform to each other [4-6]. It has been suggested that virulent FIPV arises by mutation from parental FECV in the individual, persistently infected host $[4,7,8]$. It is not yet clear which alterations in the FCoV genome are responsible for the generation of FIPV from FECV [3,6].

FIP occurs worldwide and is ubiquitous in virtually all cat populations [6]. The disease was reported as a major factor of kitten mortality in UK [9] and it is currently one of the leading infectious diseases causing death among young cats from shelters and catteries [6].

The first case of FIP in Malaysia was reported in 1981 [10] and the feature of cats with FIP were described in a 


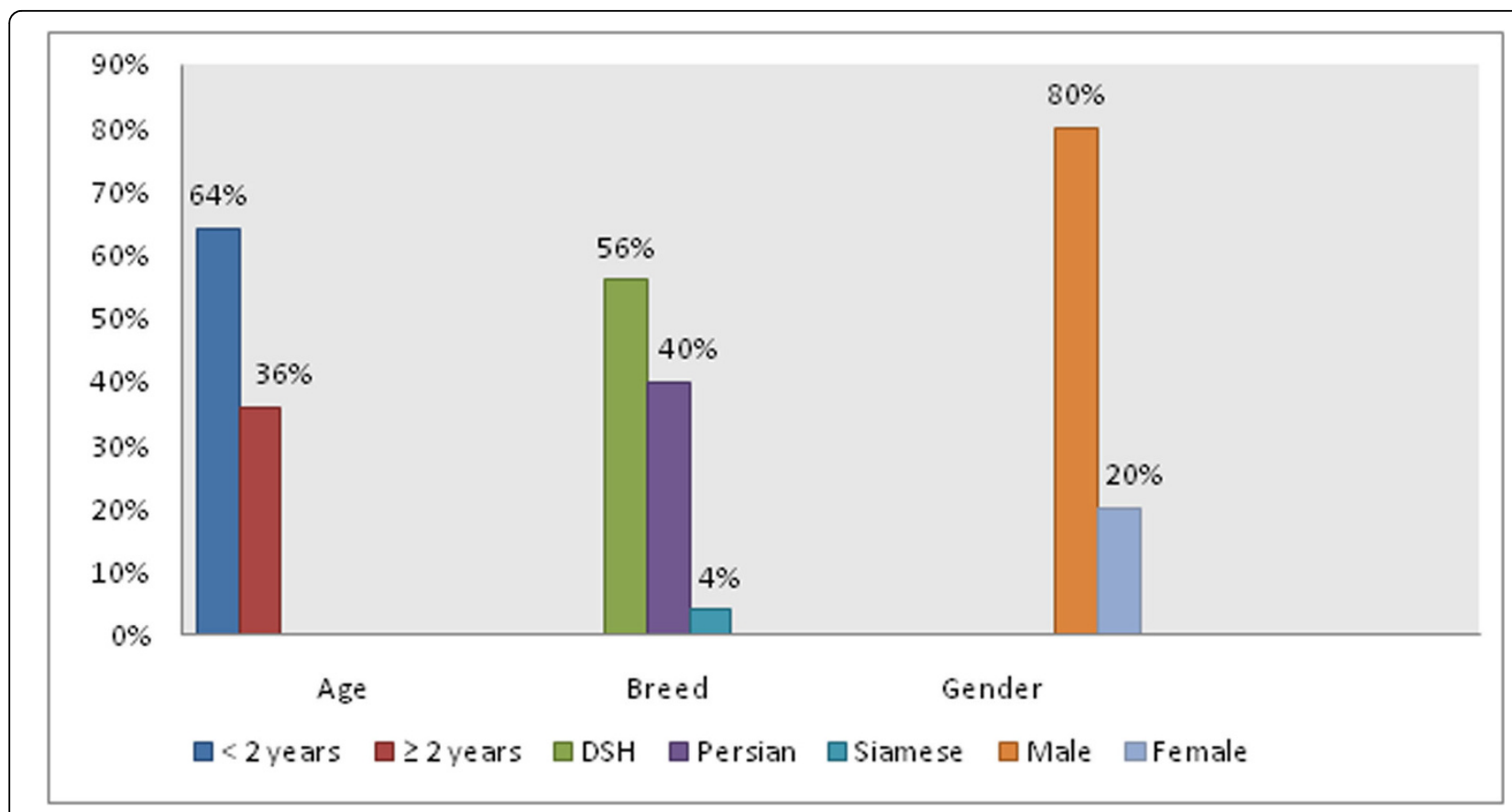

Figure 1 Distribution of feline coronavirus positive cats categorized by age, breed and gender. DSH: Domestic Short Hair

retrospective study [11]. Antibodies against FCoVs were found in $100 \%$ of cats living in Malaysian catteries [12] and the virus was detected in $84 \%$ of healthy cats using RT-PCR [13]. In present study, a conserved region of 3'untranslated region (3'UTR) is used to detect FCoV and determine the descriptive distribution and phylogeny of local isolates in FIP-suspected cats.

Abdominal fluids and/or tissue samples of 28 cats suspected of having the effusive form of FIP were obtained from the University Veterinary Hospital, Universiti Putra Malaysia (UVH-UPM) over the period of three years (2007-2009). Ascitic fluids were diluted 1:10 in phosphate buffer solution (PBS), aliquoted and stored at $-70^{\circ} \mathrm{C}$ until used. Organ samples were homogenized in 1:10 of PBS. Insoluble components were removed by centrifugation for $10 \mathrm{~min}$ at $3000 \mathrm{~g}$ and the supernatant fraction was collected and kept at $-70^{\circ} \mathrm{C}$. Two FCoV reference strains (FECV 79-1683; ATCC ${ }^{\circledR}$ No.VR-989 $^{\text {ma }}$ and FIPV79-1146; ATCC No. VR-216 ${ }^{\text {mM }}$ ) were used for RT-PCR optimization. Virus stocks were propagated in confluent Crandell Feline Kidney cells. The viruses were harvested when the infected cells showed $80 \%$ cytopathic effects. The virus suspension was freezed-thawed three times and stored at $-70^{\circ} \mathrm{C}$ until used.

RNA was extracted from the infected cell culture supernatants and clinical samples using TRIZOL ${ }^{\circ}$ Reagent (Invitrogen, Carlsbad, California, USA) according to the manufacturer's instructions. The partial 3'UTR was amplified by RT-PCR using previously described primers [7]. One-step RT-PCR was performed using Access RT-PCR System and RNasin ${ }^{\circledR}$ Ribonuclease Inhibitor (Promega, Madison, Wisconsin, USA). The

Table 1 Statistical analysis of feline infectious peritonitis suspected cats tested for feline coronavirus (FCoV) by RT-PCR assay.

\begin{tabular}{|c|c|c|c|c|c|c|c|}
\hline & Criteria & No. of tested cats & No. of FCoV-positive cats & FCoV-positivity (\%) & Odds & Odds Ratio & Confidence Interval \\
\hline \multirow[t]{2}{*}{ Age } & $<2$ years & 17 & 16 & 94 & 16 & 3.5556 & 0.2816 to 44.886 \\
\hline & $\geq 2$ years & 11 & 9 & 82 & 4.5 & & \\
\hline \multirow[t]{3}{*}{ Breed } & $\overline{\mathrm{DSH}}$ & 17 & 14 & 82 & 4.67 & * & * \\
\hline & Persian & 10 & 10 & 100 & * & * & * \\
\hline & Siamese & 1 & 1 & 100 & * & * & * \\
\hline \multirow[t]{2}{*}{ Gender } & Male & 21 & 20 & 95 & 20 & 8 & 0.5985 to 106.9411 \\
\hline & Female & 7 & 5 & 71 & 2.5 & & \\
\hline
\end{tabular}

DSH: Domestic Short Hair

* Insufficient number of cats to allow statistical calculations. 
Table 2 List of feline coronavirus isolates and strains included in the sequence and phylogenetic analysis.

\begin{tabular}{|c|c|c|c|c|}
\hline No. & Isolate/Strain & Accession No. & Origin & Reference \\
\hline 1 & UPM1C/07 & FJ897745 & Malaysia & This paper \\
\hline 2 & UPM2C/07 & FJ897746 & Malaysia & This paper \\
\hline 3 & UPM3C/07 & FJ897747 & Malaysia & This paper \\
\hline 4 & UPM4C/08 & FJ897748 & Malaysia & This paper \\
\hline 5 & UPM5C/08 & FJ897749 & Malaysia & This paper \\
\hline 6 & UPM6C/08 & FJ897750 & Malaysia & This paper \\
\hline 7 & UPM7C/09 & FJ897751 & Malaysia & This paper \\
\hline 8 & UPM8C/09 & FJ897752 & Malaysia & This paper \\
\hline 9 & UPM9C/09 & FJ897753 & Malaysia & This paper \\
\hline 10 & UPM10C/09 & FJ897754 & Malaysia & This paper \\
\hline 11 & UPM28C/08 & GQ233036 & Malaysia & [19] \\
\hline 12 & UPM29C/08 & GQ233037 & Malaysia & [19] \\
\hline 13 & UPM30C/09 & GQ233038 & Malaysia & [19] \\
\hline 14 & UPM31C/09 & GQ233039 & Malaysia & [19] \\
\hline 15 & UU10 & FJ938059 & Netherlands & Unpublished \\
\hline 16 & UU15 & FJ938057 & Netherlands & Unpublished \\
\hline 17 & UU11 & FJ938052 & Netherlands & Unpublished \\
\hline 18 & UU9 & FJ938062 & Netherlands & Unpublished \\
\hline 19 & UU3 & FJ938061 & USA & Unpublished \\
\hline 20 & UU2 & FJ938060 & USA & Unpublished \\
\hline 21 & RM & FJ938051 & USA & Unpublished \\
\hline 22 & UCD11b-2b & FJ917535 & USA & Unpublished \\
\hline 23 & UCD11b-2a & FJ917534 & USA & Unpublished \\
\hline 24 & UCD 1 1b-1b & FJ917533 & USA & Unpublished \\
\hline 25 & UCD11b-1a & FJ917532 & USA & Unpublished \\
\hline 26 & UCD11a-1b & FJ917531 & USA & Unpublished \\
\hline 27 & UCD11a-1a & FJ917530 & USA & Unpublished \\
\hline 28 & UCD17 & FJ917527 & USA & Unpublished \\
\hline 29 & UCD14 & FJ917524 & USA & Unpublished \\
\hline 30 & UCD13 & FJ917523 & USA & Unpublished \\
\hline 31 & UCD5 & FJ917522 & USA & Unpublished \\
\hline 32 & UCD12 & FJ917521 & USA & Unpublished \\
\hline 33 & UCD11b & FJ917520 & USA & Unpublished \\
\hline 34 & UCD11a & FJ917519 & USA & Unpublished \\
\hline 35 & Black & EU186072 & USA & {$[3]$} \\
\hline 36 & NTU2/R/2003 & DQ160294 & Taiwan & Unpublished \\
\hline 37 & UU16 & FJ938058 & Netherlands & Unpublished \\
\hline 38 & UU5 & FJ938056 & Netherlands & Unpublished \\
\hline 39 & UU8 & FJ938055 & Netherlands & Unpublished \\
\hline 40 & UU7 & FJ938053 & Netherlands & Unpublished \\
\hline 41 & UCD18b & FJ917529 & USA & Unpublished \\
\hline 42 & UCD18a & FJ917528 & USA & Unpublished \\
\hline 43 & UCD16 & FJ917526 & USA & Unpublished \\
\hline 44 & UCD15a & FJ917525 & USA & Unpublished \\
\hline 45 & DF-2 & DQ286389 & USA & Unpublished \\
\hline 46 & C1Je & DQ848678 & UK & Unpublished \\
\hline 47 & NTU156/P/2007 & GQ152141 & Taiwan & Unpublished \\
\hline 48 & UU4 & FJ938054 & Netherlands & Unpublished \\
\hline 49 & 79-1146 & DQ010921 & USA & {$[21]$} \\
\hline 50 & Wellcome & X90571 & Netherlands & [22] \\
\hline
\end{tabular}

Table 2: List of feline coronavirus isolates and strains included in the sequence and phylogenetic analysis. (Continued)

\begin{tabular}{lllll}
\hline 51 & UCD1 & X90575 & USA & {$[22]$} \\
52 & UCD & X90574 & USA & {$[22]$} \\
53 & TN406 & X90570 & Netherlands & {$[22]$} \\
54 & UCD3a & FJ943761 & USA & Unpublished \\
55 & UCD2 & X90576 & USA & {$[22]$} \\
56 & Dahlberg & X90572 & Netherlands & {$[22]$} \\
57 & UCD3 & X90577 & USA & {$[22]$} \\
58 & UCD4 & X90578 & USA & {$[22]$} \\
59 & NOR15 & X90573 & Netherlands & {$[22]$} \\
60 & UCD12-1 & FJ943766 & USA & Unpublished \\
61 & UCD6-1 & FJ943772 & USA & Unpublished \\
62 & $79-1683$ & X66718 & USA & {$[23]$} \\
\hline
\end{tabular}

reaction was optimized on a thermal cycler (MJ Research, Waltham, Massachusetts, USA). PCR products of $223 \mathrm{bp}$ were analyzed using electrophoresis on a $2 \%$ agarose gel, stained with ethidium bromide and observed under UV light. PCR products of 10 positive cases were selected randomly, purified using PCR SV protocol (GENEALL ${ }^{\circ}$, Seoul, South Korea) and sequenced in both direction with the primers (Medigene, Selangor, Malaysia).

Data analysis was performed using Statistical Tables Calculator, which is available online at http://faculty.vassar.edu/lowry/odds2x2.html. Age, breed and gender differences were compared by calculating positivity rate, odds and $95 \%$ confidence intervals.

The RT-PCR assay amplified the target band in 25 out of 28 cats' samples (89\%). Although, the PCR results must be interpreted in conjunction with clinical or pathological findings, detection of the virus in FIP-suspected cats may be useful to confirm FIP. Since FCoVs are ubiquitous in cats with high seroprevalence [5,6,12], PCR provides the obvious advantage over serology by directly detecting FCoV genome rather than documenting a previous immune system encounter with the coronavirus. The primers of this PCR assay were chosen from a highly conserved region of 3'UTR of the FCoV genome to detect most, if not all of the FCoV strains. The usefulness of these primers for a general screening test has been reported previously [14-16].

FCoV-positivity rate in cats younger than two years old $(64 \%)$ was higher than older cats, but they are not significant. However, the result is consistent with other studies demonstrating higher incidence of FIP in cats below 2 years of age $[5,11,14]$ and agree with the fact that FIP is a disease of young cats. Typical clinical cases are first appear during the postweaning period, but most deaths from FIP occur in cats 3-16 months of age [6].

Most of the FCoV-positive cats in our study were males (80\%). Higher incidence of FIP among males was 


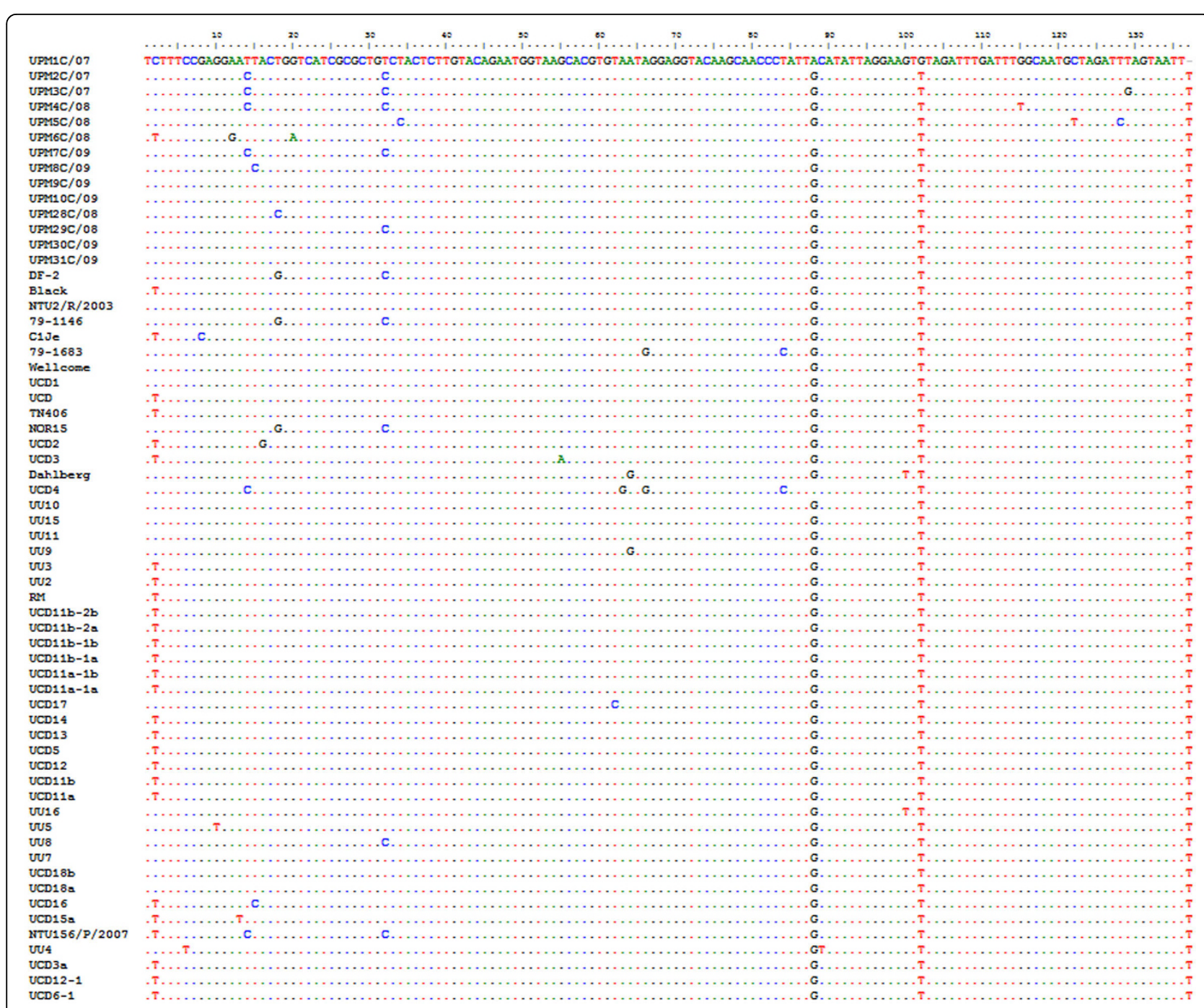

Figure 2 Comparison of partial sequence of 3'UTR of Malaysian isolates and reference strains of feline coronaviruses. Multiple alignments were performed using ClustalW Multiple alignment (Bioedit version 7.0.9). The sequences of the primers were removed from the alignment. Dots indicate identity.

previously reported $[14,17,18]$. As the pathogenesis of the disease is still not fully understood, the relation of gender and incidence of FIP is not clear.

About 56\% of FCoV-positive cases were DSH, 40\% Persian, and $4 \%$ Siamese cats. In the present study, the majority of cats (96\%) diagnosed with FIP were DSH or Persian. This finding is in accordance with a previous report on FIP in Malaysia showing that $69.7 \%$ and $27.3 \%$ of cats diagnosed with FIP were DSH and Persian cats, respectively [11]. However, these studies did not conclude that these two breeds were more susceptible to FIP because of limited variation in cat breeds presented at UVH-UPM and lack of clinical cases of FIP in different breeds in Malaysia. Furthermore, in a study on the prevalence of FIP in specific cat breeds, DSH and Persian cats were at low risk compared to others [18]. Age, breed and gender distribution in FCoV-positive cats are shown in Figure 1 and statistical analysis is summarized in Table 1.

Out of 25 PCR positive cases, 10 isolates were selected for further sequence analyses. All 10 field isolates designated as UPM1C/07 to UPM10C/09 with accession no. FJ897745 to FJ897754, respectively were deposited in the GeneBank (Table 2). These sequences were aligned with published sequences of FCoV using ClustalW Multiple alignment (Bioedit version 7.0.9). The sequences of four Malaysian FCoV isolates which have been isolated from healthy cats in a previous study [19] were also included in the alignment (Table 2). Homology matrix and phylogenetic trees were constructed using Neighbor-Joining method (Bioedit) and TreeTop-Phylogenetic Tree Prediction (GeneBee-Molecular Biology Server 


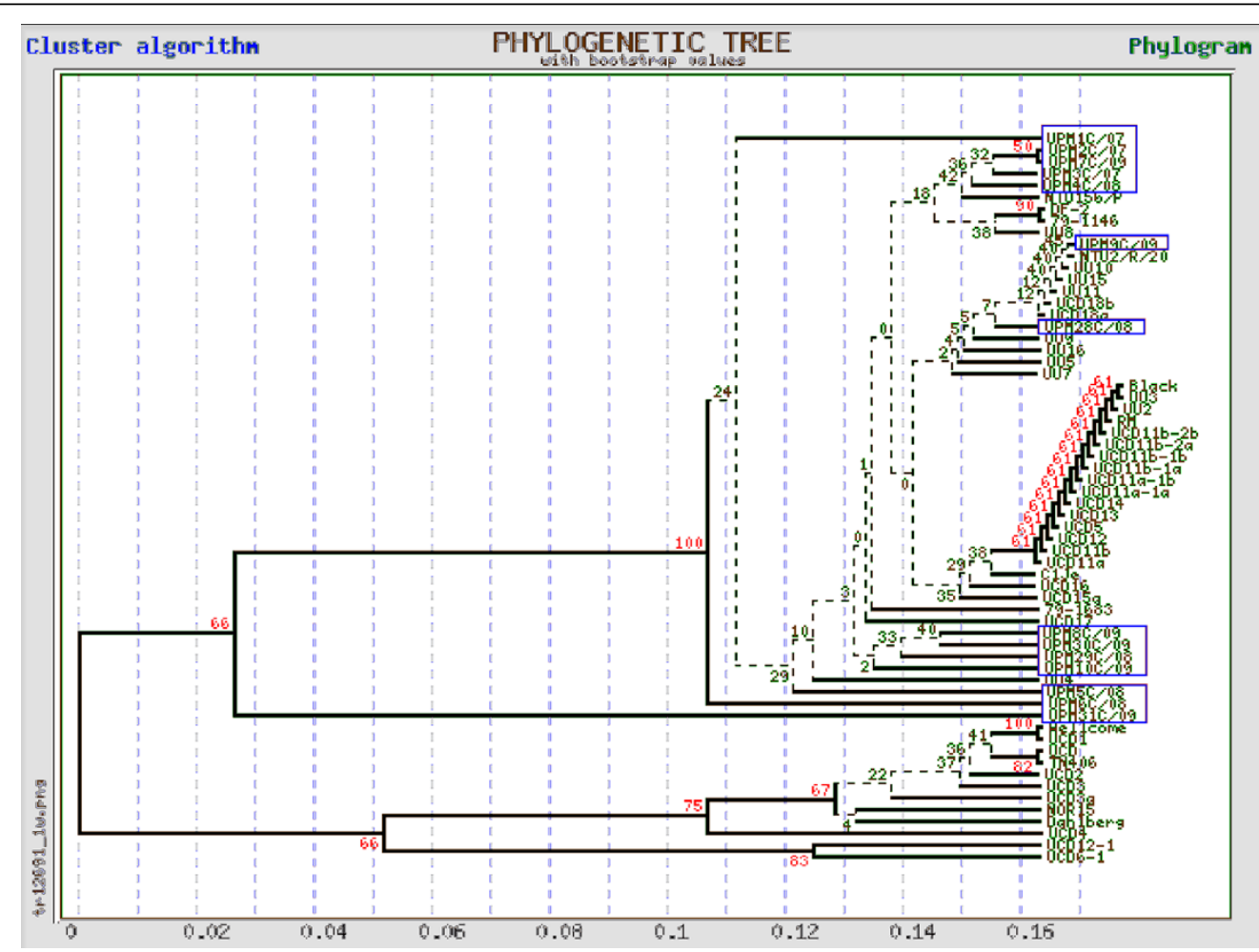

Figure 3 Phylogenetic tree based on partial sequence of feline coronaviruses. Malaysian isolates are marked by frames and categorized in one main cluster. The tree constructed by Tree Top-Phylogenetic Tree Prediction (GeneBee - Molecular Biology Server). The tree is displayed in PHYLIP format with bootstrap values.

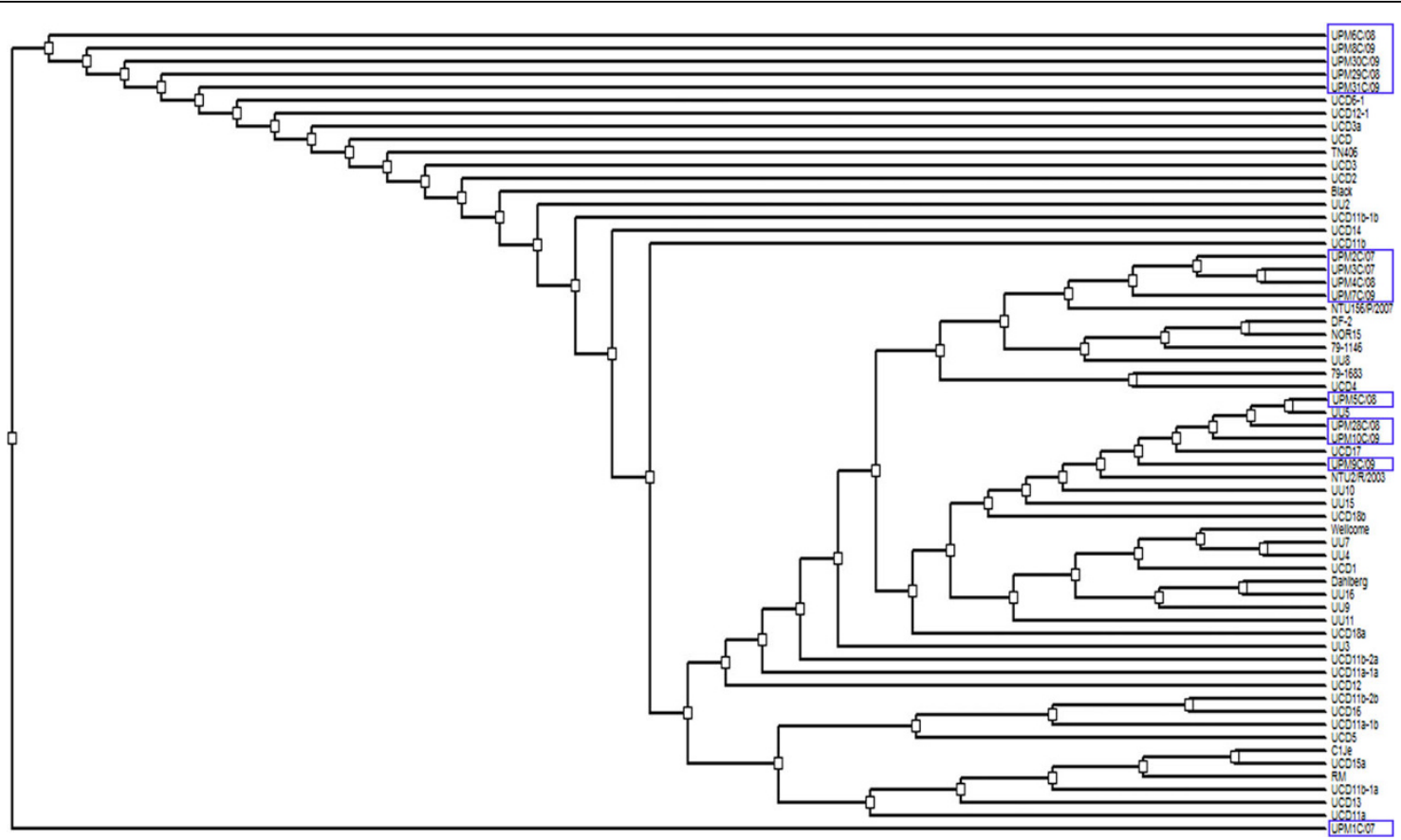

Figure 4 Neighbor phylogenetic tree of feline coronavirus (FCoV) strains and isolates. Partial sequences of FCoVs were subjected to DNADist version 3.5c and the result showed as a neighbor-joining algorithm (Bioedit version 7.0.9). Malaysian isolates are marked by frames. 
available at http://www.genebee.msu.su). The phylogenetic trees were displayed in PHYLIP format including bootstrap values.

The sequences of ten local isolates showed 96\% homology and when compared to published sequences of $\mathrm{FCoV}$, the homology decreased to $93 \%$. The homology between partial sequences of FCoV isolates from Malaysia were higher than those from different geographical origin (32 strains from USA, 13 strains from Netherlands, two strains from Taiwan, and one strain from UK). These findings support previous observations showing a correlation between different FCoV biotypes with similar geographic background [8].

Multiple sequence alignment showed a few point mutations and single-nucleotide deletions in the sequences of local isolates (Figure 2). These findings indicate single nucleotide polymorphisms (SNPs) in FCoVs as described previously $[6,20]$. No particular pattern of mutation or deletion was found in this part of FCoVs genome.

Phylogenetic tree constructed by cluster algorithm showed that the sequences were genetically separated in two distinct clusters; all local sequences fell into one main cluster and suggested they may derived from a common ancestor (Figure 3). However, a whole genome sequence is needed to determine genetic pattern of Malaysian FCoVs. Phylogenetic tree constructed by neighbor-joining method showed the phylogenetic relations of the sequences in an unrooted-tree algorithm (Figure 4).

In conclusion, the present study indicated that males and young cats are more likely to be diagnosed with FIP. The homology of partial sequences of 3'UTR of FCoV isolates in Malaysia was shown to be higher than those from the other regions.

\section{Acknowledgements}

The authors would like to thank the staffs of the University Veterinary Hospital and cat owners who participate in this project. The study was funded by MOSTI project no. 02-01-04-SF0485: Development of a rapid test for diagnosis of feline coronavirus.

\section{Author details}

'Department of Veterinary Pathology and Microbiology, Faculty of Veterinary Medicine, Universiti Putra Malaysia, 43400 UPM Serdang, Selangor, Malaysia. ${ }^{2}$ Department of Clinical Studies, Faculty of Veterinary Medicine, Universiti Putra Malaysia, 43400 UPM Serdang, Selangor, Malaysia. ${ }^{3}$ University Veterinary Hospital, Faculty of Veterinary Medicine, Universiti Putra Malaysia, 43400 UPM Serdang, Selangor, Malaysia.

\section{Authors' contributions}

SSA designed and coordinated the study and helped in draft correction. SSH carried out the molecular studies, performed the RT-PCR assay and sequence analysis and drafted the manuscript. MHB, ARO and NAZ participated in the sequence analysis and proof reading. LSF, NAR, HA and SHSH participated in the collecting of clinical samples. MAHI helped in lab works. All authors read and approved the final manuscript.

\section{Competing interests}

The authors declare that they have no competing interests.

Received: 2 September 2009

Accepted: 6 January 2010 Published: 6 January 2010

\section{References}

1. Lai MM, Perlman S, Anderson L: Coronaviridae. Fields Virology Philadelphia: Lippincott Williams \& WilkinsKnipe DM, Howley PM, 5 2007, 1305-1335.

2. Vijaykrishna D, Smith GJ, Zhang JX, Peiris JS, Chen H, Guan Y: Evolutionary insights into the ecology of coronaviruses. J Virology 2007, 81:4012-20.

3. Tekes G, Hofmann-Lehmann R, Stallkamp I, Thiel V, Thiel H: Genome organization and reverse genetic analysis of a type I feline coronavirus. J Virology 2008, 82:1851-1859.

4. Pedersen NC, Boyle JF, Floyd K, Fudge A, Barker J: An enteric coronavirus infection of cats and its relationship to feline infectious peritonitis. Am J Vet Res 1981, 42:368-377.

5. Hartmann K: Feline Infectious Peritonitis. Veterinary Clinics of North America Small Animal Practice, Advances in Feline Medicine Philadelphia: W.B. Saunders CompanyRichards RJ 2005, 39-79.

6. Pedersen NC: A review of feline infectious peritonitis virus infection: 1963-2000. J Feline Med Surg 2009, 11:225-258

7. Herrewegh AA, De Groot RJ, Cepica A, Egberink HF, Horzinek MC, Rottier PJ: Detection of feline coronavirus RNA in feces, tissues, and body fluids of naturally infected cats by reverse transcriptase PCR. J Clin Micro 1995, 33:684-689.

8. Vennema H, Poland A, Foley J, Pedersen N: Feline infectious peritonitis virus arise by mutation from endemic feline enteric coronaviruses. Virology 1998, 243:150-157.

9. Cave TA, Thompson H, Reid SW, et al: Kitten mortality in the United Kingdom: a retrospective analysis of 274 histopathological examinations (1986 to 2000). Vet Rec 2002, 151:497-501.

10. Wong WT, Cheng BY, Lee JS: Feline infectious peritonitis - two case reports. Kajian Vet Malaysia 1983, 15:30-35.

11. Ahmad AA, Arshad SS, Lee WW, Hassan L, Nor-Alimah AR, Siti-Aminah Y, Habibah A: Retrospective examination of feline infectious peritonitis cases presented to University Veterinary Hospital, Universiti Putra Malaysia (UVH-UPM) between 2004-2007. Proceedings of the 19th Veterinary Association Malaysia Congress, 3-5 August 2007 170-172.

12. Arshad SS, Lee WW, Hassan L, Kamarudin AM, Siti-Farawahida AW Cheng NB: Serological survey of catteries for cats infected with feline coronavirus. J Vet Malaysia 2004, 17:19-22

13. Sharif S, Arshad SS, Hair-Bejo M, Omar AR, Zeenathul NA, Hafidz MA Prevalence of feline coronavirus in two cat populations in Malaysia. $J$ Feline Med Surg 2009, 11:1031-1034

14. Benetka V, Kubber-Heis A, Kolodziejek J, Nowotny N, Hofmann-Parisot M, Mostl K: Prevalence of feline coronavirus types I and II in cats with histopathologically verified feline infectious peritonitis. Vet Micro 2004, 99:31-42.

15. Lin CN, Su BL, Huang HP, Lee JJ, Hsieh MW, Chueh LL: Field strain feline coronaviruses with small deletions in ORF7b associated with both enteric infection and feline infectious peritonitis. J Feline Med Surg 2009 11:413-419.

16. Duarte A, Veiga I, Tavares L: Genetic diversity and phylogenetic analysis of feline coronavirus sequences from Portugal. Vet Micro 2009, 138:163168

17. Potkay S, Bacher JD, Pitts TW: Feline infectious peritonitis in a closed breeding colony. Lab Anim Sci 1974, 24:279-289.

18. Pesteanu-Somogyi LD, Radzai C, Pressler BM: Prevalence of feline infectious peritonitis in specific cat breeds. J Feline Med Surg 2006, 8:1-5.

19. Sharif S, Arshad SS, Hair-Bejo M, Omar AR, Zeenathul NA, Hafidz MA: Phylogenetic analysis of feline coronavirus isolates from healthy cats in Malaysia. Proceedings of the 8th Malaysia Genetics Congress; 4-6 August Genting, Malaysia 2009.

20. Battilani M, Coradin T, Scagliarini A, Ciulli S, Ostanello F, Prosperi S, Morganti L: Quasispecies composition and phylogenetic analysis of feline coronaviruses (FCoVs) in naturally infected cats. FEMS Immunol Med Micro 2003, 39:141-147. 
21. Dye C, Siddell SG: Genomic RNA sequence of feline coronavirus strain FIPV WSU-79/1146. J Gen Virol 2005, 86:2249-2253.

22. Herrewegh AA, Vennema H, Horzinek MC, Rottier PJ, de Groot RJ: The molecular genetics of feline coronaviruses: comparative sequence analysis of the ORF7a/7b transcription unit of different biotypes. Virology 1995, 212:622-631.

23. Vennema H, Rossen JW, Wesseling J, Horzinek MC, Rottier PJ: Genomic organization and expression of the $3^{\prime}$ end of the canine and feline enteric coronaviruses. Virology 1992, 191:134-140.

doi:10.1186/1751-0147-52-1

Cite this article as: Sharif et al: Descriptive distribution and

phylogenetic analysis of feline infectious peritonitis virus isolates of Malaysia. Acta Veterinaria Scandinavica 2010 52:1.

Publish with Biomed Central and every scientist can read your work free of charge

"BioMed Central will be the most significant development for disseminating the results of biomedical research in our lifetime. " Sir Paul Nurse, Cancer Research UK

Your research papers will be:

- available free of charge to the entire biomedical community

- peer reviewed and published immediately upon acceptance

- cited in PubMed and archived on PubMed Central

- yours - you keep the copyright 